\title{
MR of the liver: from breakthrough to clinical application
}

\section{Carlo Bartolozzi}

Department of Diagnostic and Interventional Radiology, University of Pisa, Via Paradisa 2 Cisanello, 56125 Pisa, Italy

Liver represents, for the Radiologist, a topic of extraordinary interest, considering the multiple and very different pathological conditions that may affect the organ; moreover, a tremendous development of new imaging techniques, applicable in this specific field of interest, has taken place during these last years.

Nowadays, there is no doubt that among the several tools dedicated to the study of liver parenchyma, MR plays a key role in management of liver pathologies, especially thanks to the very rapid growth of new methods, to their prompt validation and the consequent exploitation in the clinical environment; so that MR is actually considered of the utmost importance for the diagnosis and therapeutic decisions in a patient suffering from liver disease.

Particularly, the actual technical approach is based on the application of very fast sequences, that perfectly allow the depiction of lesions' vascular supply.

Moreover, the development of diffusion-weighted (DW) MR imaging as well as the introduction of hepatobiliary contrast agents represent a link to a new approach in the evaluation of liver pathologies.
In fact, DW imaging enables a quali-quantitative assessment of tissue diffusivity, thus suggesting yet on baseline study, not only the diagnosis of a native lesion, but also a clue for a different evaluation of tumor response to treatments.

Furthermore, hepatobiliary contrast agents can help in exploring the sophisticated processes that take place during the development of nodular malignancy, thus permitting to better stratify patients on the basis of the aggressiveness of their disease.

It is thus my great honor to introduce this Feature Section in which six leading experts focus on six different topics, following a sort of "fil rouge", that moves from applicable breakthroughs, in terms of new acquisition techniques and new contrast media, up to the relevance of the technique within the clinical setting, represented by the reliable differential diagnosis among nonneoplastic and neoplastic lesions of the liver.

Correspondence to: Carlo Bartolozzi; email: bartolozzi@med.unipi.it 